\title{
Interacción de la leptina y el hueso en sujetos obesos. Protección vs. fragilidad
}

\author{
Ricardo Rosero ${ }^{1}$, Ariana Sierra ${ }^{2}$, Adriana Medina ${ }^{3}$
}

${ }^{1}$ Médico Internista y Endocrinólogo, Departamento de Endocrinología, Hospital San Jose Infantil, Bogotá

${ }^{2}$ Medica Internista y Endocrinóloga, Departamento de Endocrinología Hospital San Jose, Bogotá

${ }^{3}$ Medica Internista y Endocrinóloga, Departamento de Endocrinología Hospital San Jose, Bogotá

\section{Resumen}

$\mathrm{L}$ a osteoporois y la obesidad son dos de los desórdenes más frecuentes e importantes actualmente en endocrinología, con una prevalencia en aumento; ambas entidades alteran la configuración y mecánica del cuerpo y comparten características genéticas, con un progenitor común. Con el envejecimiento, la relación entre obesidad y baja densidad ósea se afianza, produciendo predominio de la adiposidad y osteoclastogénesis, que altera la función del osteoblasto, por el efecto protagónico de la leptina como modulador de la diferenciación de células mesenquimales a adipocitos y no a osteoblastos, lo que lleva a la expresión de la enfermedad.

En esta revisión queremos esclarecer fisiológicamente la estrecha relación entre estas dos condiciones (osteoporosis y obesidad) y establecer el papel estelar de las adipocinas en la patogenía de la osteoporosis.

Palabras claves: Leptina, Obesidad, Osteoporosis, Densidad ósea.

\section{Introducción}

Durante esta última década, la obesidad ha tenido un cambio radical, ya que dejó de ser un factor de riesgo para ser aceptada y entendida como una enfermedad. Esta enfermedad tiene una gran importancia a nivel sistémico, puesto que afecta, casi sin exclusión, todos los órganos de la economía corporal, generando patologías que se asocian con una alta tasa de morbilidad y mortalidad. Lo llamativo es que a pesar de que la obesidad, como factor etiológico, está asociada a efectos mórbidos, el hueso parece beneficiarse de ella, como lo afirma Iwaniec et al. en su publicación en la que se intenta demostrar cómo el sobrepeso y la obesidad se relacionan con una mayor densidad mineral ósea ${ }^{(1)}$.
Una vez nos detenemos a estudiar la obesidad como un gran fenómeno, en detalle, aparecen ciertos puntos claves como la alteración en las vías de señalización intracelular, receptores, citocinas, hormonas, entre otros y tal vez el eslabón que conecta todos estos efectos es la leptina.

En 1994, Zhang et al. en la universidad de Rockefeller, lograron aislar el producto del gen $o b^{(2)}$, el cual fue llamado leptina, derivado de la raíz griega Leptos, que significa delgado.

El descubrimiento de que la mayoría de los sujetos obesos humanos son resistentes o intolerantes a la leptina, hizo considerar a la leptina como un objetivo terapéutico muy prometedor para el control de la obesidad, pero sólo probó ser eficaz en pacientes que presentaban deficiencia congénita de la leptina ${ }^{(3)}$. Posteriormente y continuando los estudios sobre esta hormona tan ligada a la obesidad, apareció evidencia que relacionaba la leptina con múltiples efectos agudos y crónicos en los estados de deficiencia energética ${ }^{(4)}$. La leptina ha emergido como un posible factor responsable en gran medida de los efectos deletéreos del tejido graso sobre diferentes órganos, por ejemplo: la resistencia a la insulina a nivel del músculo periférico, la ateromatosis en el endotelio, el efecto anorexígeno a nivel del hipotálamo y el metabolismo mineral en el hueso, donde favorece la diferenciación de los precursores óseos que se enunciarán posteriormente ${ }^{(5)}$.

\section{Leptina, la protagonista}

La leptina es el prototipo de las adipocinas. Es un péptido de 167 aminoácidos con cuatro hélices y circula en forma de monómero ${ }^{(6)}$. Es uno de los productos primarios del tejido adiposo, pero también se expresa en diferentes órganos como la placenta, los ovarios, el epitelio mamario, la médula ósea y el tejido linfoide ${ }^{(7)}$.

Los niveles de leptina son producto de una secreción pulsátil y tienen un ritmo circadiano, con niveles altos en la madrugada; este ritmo se conserva en sujetos obesos y delgados, pero con la diferencia de que en el grupo de sujetos obesos hay mayor amplitud, lo que se traduce en mayores niveles séricos de leptina ${ }^{(8)}$.

Una vez secretada la leptina al torrente sanguíneo, circula parcialmente adherida a proteínas plasmáticas ${ }^{(8)}$. Los niveles 
séricos de la leptina en personas con peso normal oscilan en el rango de 1 a $15 \mathrm{ng} / \mathrm{ml}$, en individuos con índice de masa corporal mayor a $30 \mathrm{~kg} / \mathrm{m}^{2}$ (obesidad), se pueden encontrar valores mayores, la vida media es de 25 minutos, y tiene una depuración rápida con eliminación renal ${ }^{(8)}$.

La concentración de leptina refleja la cantidad de energía acumulada en el compartimiento graso ${ }^{(9)}$, con cambios dramáticos en periodo de ayuno y posprandial ${ }^{(10)}$.

La leptina se une a su receptor (ObR) localizado en el sistema nervioso central y tejido periférico. Existen por lo menos seis isoformas de receptores identificados (ObRa, ObRb, ObRc, ObRd, ObRe y ObRf) ${ }^{(2,7)}$. Estas isoformas presentan dominios homólogos extracelulares pero con diferentes dominios intracelulares $^{(7)}$ : las isoformas cortas como ObRa y ObRc tienen un papel importante en el transporte de la leptina al sistema nervioso central y las isoformas largas son responsables de la señalización ${ }^{(11)}$.

La señalización se lleva a cabo por diferentes vías. La activación de los receptores ObRb ocurre mediante una cascada de señalización de transducción a través de la activación de transcripción 3 (JAK2/STAT3), la cual es la más descrita. Esta

Figura 1. La leptina (L), al interactuar con su receptor ObRb, establece conexiones entre la superficie celular y el núcleo, en las que las cascadas de cinasas proteínicas dan lugar a la fosforilación de factores de transcripción. JAK2 fosforila proteínas con dominios SH2, estas proteínas pertenecen a tres familias diferentes: STAT3, ERK (ERK: cinasa estimulada por señales extracelulares - Vía MAPK) e IRS (principalmente el IRS-2). Las STAT3 y ERK, una vez fosforiladas, son translocadas al núcleo donde funcionan como reguladoras transcripcionales ${ }^{(14)}$.

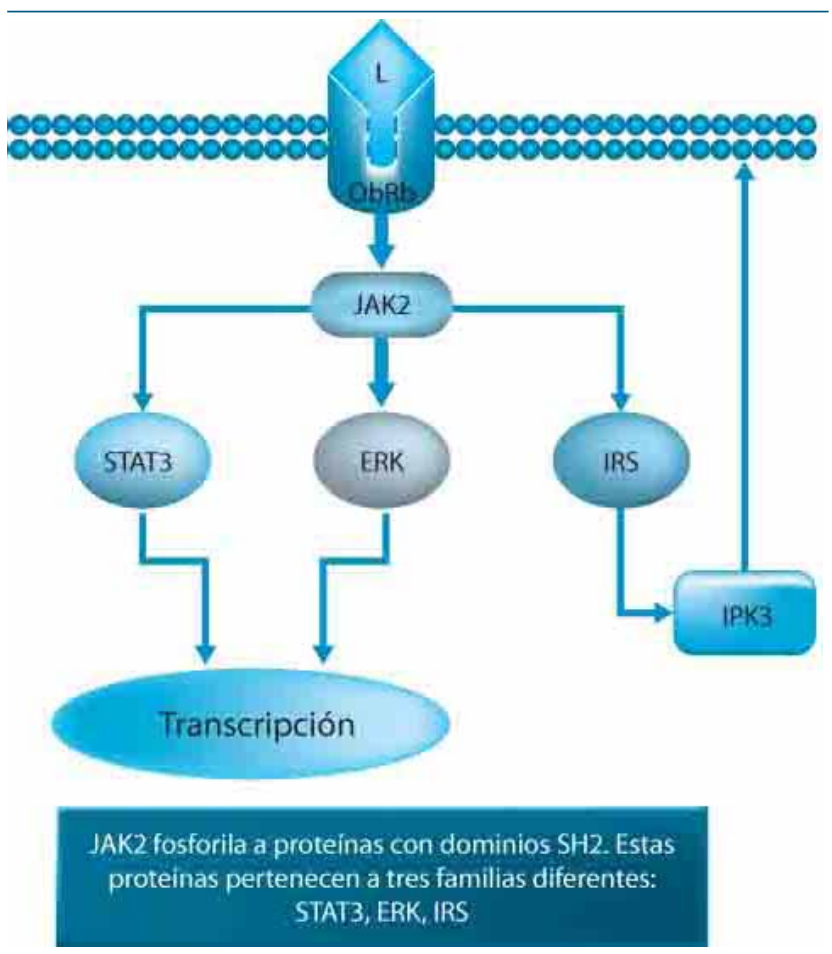

vía juega un papel importante en la homeostasis de la energía. La activación de STAT3 por la leptina induce transcripción de propiomelanocortina (POMC), un neuropéptido anorexígeno ubicado en el núcleo arcuato del hipotálamo ${ }^{(12)}$. Las alteraciones en STAT3 afectan la producción de sustancias orexígenas como el péptido relacionado con agoutí y el neuropéptido $\mathrm{Y}$ que resultan en hiperleptinemia, hiperfagia, ganancia moderada de peso e hiperinsulinemia ${ }^{(13)}$ (figura 1).

Algunos estudios sugieren que la leptina estimula la vía fosfatidilinositol 3 cinasa (IP3K), que se asocia al tejido adiposo, hígado, músculo y células cancerígenas en humanos ${ }^{(15,16)}$. La activación de IP3K ocurre a través de sustratos del receptor de insulina (IRS), particularmente IRS-2 ${ }^{(15)}$.

La activación de IP3K en el hipotálamo contribuye a la acción anorexígena de la leptina y a la pérdida de peso. Al igual que STAT3, la IP3K activa las neuronas POMC por la activación

Figura 2. Al unirse la leptina a su receptor de membrana ObRb se produce dimerización y autofosforilación del receptor, evento que permite el anclaje de proteínas Janus-tirosina-cinasa JAK2 al dominio intracelular del receptor. Las ERK, una vez fosforiladas, se translocan al núcleo, fosforilando factores de transcripción para la expresión de genes que regulan la proliferación, diferenciación y supervivencia celular en condiciones normales. Si se altera la señalización ejercida por la leptina (como en casos de resistencia a la leptina), al fallar la fosforilación de la ERK, se desencadenará apoptosis celular ${ }^{(14,22)}$.

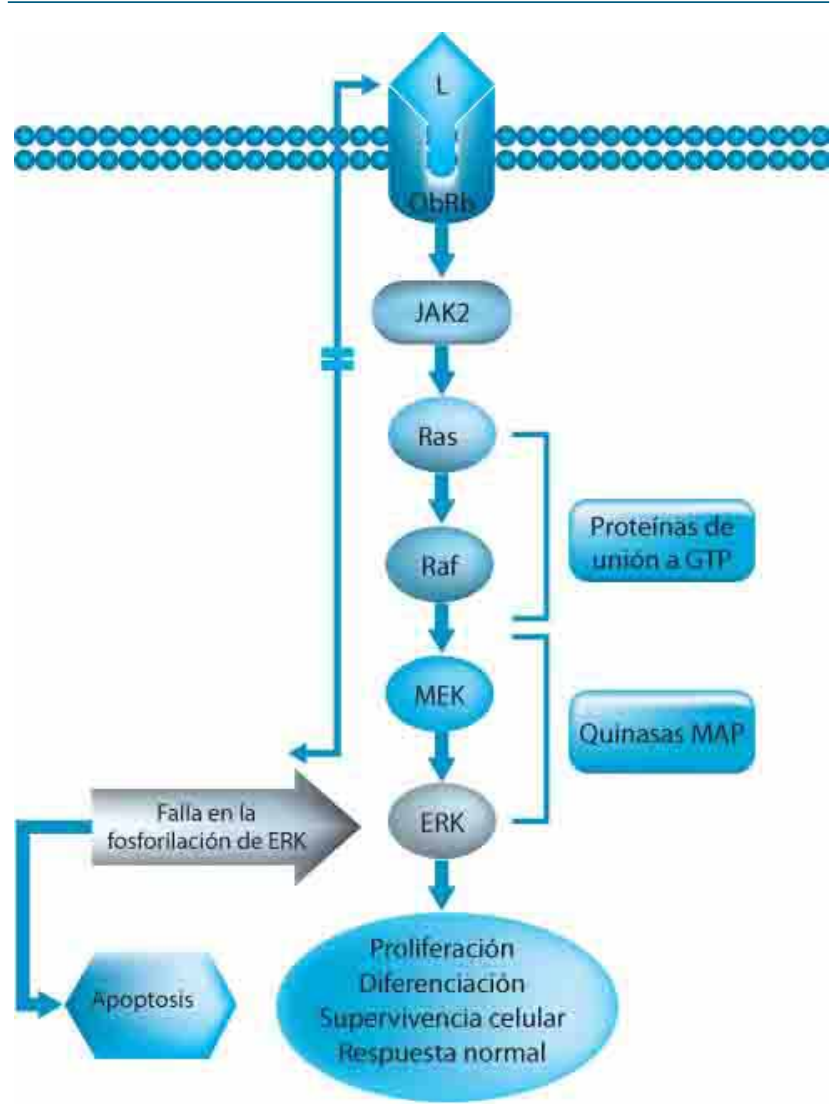


de los canales de potasio dependientes de ATP y canales de calcio $^{(15)}$. Igual que la insulina, el apetito suprime los efectos de la leptina. La vía insulina-IP3K hiperosmolariza las neuronas POMC, generando menor sensibilidad a la leptina, y eso podría ser un mecanismo común concomitante entre la resistencia a la leptina e insulina en la obesidad ${ }^{(17)}$.

Las proteínas cinasas mitogénicas-activadoras (MAPK) son un grupo de cinasas que controlan un gran número de procesos celulares. La leptina es capaz de activar la cascada MAPK en dos diferentes vías: una dependiente, a través de la vía de fosforilación de la tirosina en los receptores asociados a la activación de JAK2, y otra independiente de la fosforilación de receptores. Sin embargo, en ambas vías, la señalización requiere un dominio catalítico intacto de SHP2.

Se ha reportado que alteraciones en la fosforilación causan falla en la activación de $\operatorname{ERK}^{(18)}$. La activación de la vía de señalización tiene relación con las células precursoras del linaje de osteoblastos, en la cual se ha demostrado cómo la leptina induce apoptosis del osteoblasto a través de la cascada MAPK, con la activación de la vía ERK1/2, que a su vez activa la fosfolipasa citosólica A, que lleva a la liberación del citocromo $\mathrm{C}$ $y$, finalmente, la inducción de la caspasa 3 y la caspasa $9^{(19-21)}$. (figura 2).

El papel de la leptina en el metabolismo óseo es evidente de acuerdo con lo observado en múltiples estudios, afectándolo a través de la vía central y periférica. La leptina, a nivel central, regula la formación cortical del hueso, mediante la activación simpática en los núcleos ventrolaterales del hipotála$\mathrm{mo}^{(23,24)}$. De igual forma, existe una relación entre la leptina y la expresión de neuromedina $U$, la cual, al parecer, disminuye el hueso tanto cortical como trabecular ${ }^{(23,25)}$. Otra acción a nivel central mediada por la leptina es la inhibición y reducción de la síntesis de neuronas serotoninérgicas, que juegan un papel aún no aclarado sobre el metabolismo del hueso ${ }^{(26)}$.

A nivel periférico, la leptina interactúa con las células estromales de la médula ósea, osteoblastos y osteoclastos para incrementar la masa ósea. La leptina incrementa la expresión de genes osteogénicos en las células estromales y las dirige a la formación de hueso adipogénico ${ }^{(23,27)}$, así mismo, la leptina puede inhibir la generación de osteoclastos y la resorción ósea por mecanismos locales confinados a los linajes hematopoyéticos, los cuales estimulan las células estromales que incrementan la expresión de osteoprotegerina y disminuyen el ligando RANK, llevando a la disminución de osteoclastogénesis. Las perturbaciones intrínsecas y extrínsecas de la actividad de la leptina en el hueso del paciente obeso producen un cambio en la diferenciación estromal a favor de líneas adipocitarias, que terminan directamente en la reducción de la formación y reducción de la resorción óseas ${ }^{(28)}$.

La mayoría de los pacientes obesos presentan niveles de leptina aumentados, por resistencia o por tolerancia ${ }^{(9)}$. En estos pacientes se observa, primero, alteración en el transporte

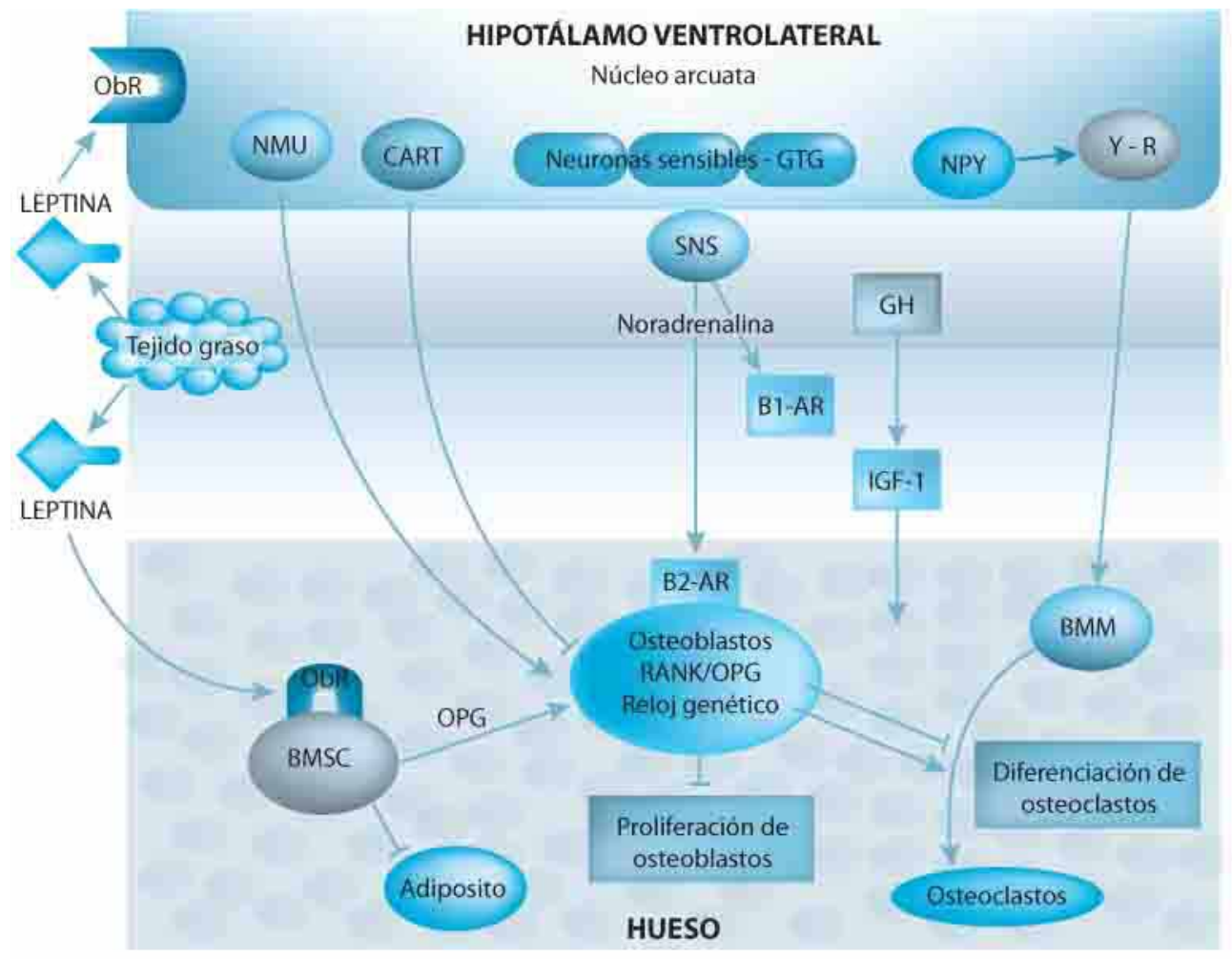

Diagrama obtenido de la publicación de Leptina \& Bone. Minireview, The Effect of Letpin on Bone $^{(29)}$ en donde se explican las diferentes vías en las que la leptina tiene acciones indirectas, en el sistema nervioso central y a nivel periférico, directamente en el hueso. 
de la leptina a través de la barrera hemato-encefálica, debido a una saturación del transportador por hiperleptinemia, el cual está asociado con obesidad ${ }^{(30)}$. Adicionalmente a la hiperleptinemia, la hipertrigliceridemia afecta el transporte de lepti$\mathrm{na}^{(31)}$. También existen alteraciones en la señalización, secundarias a la hiperleptinemia, como son el efecto del supresor de la señalización de citocinas 3 (SOCS3), que es el mayor inhibidor de la señalización de la leptina. La vía JAK2/STAT3 induce SOCS3, el cual inhibe la fosforilación y activación de JAK2 en el residuo de tirosina del receptor $\mathrm{ObRb}^{(31)}$. La proteína tirosina fosfatasa (SHP2) también está activada por la leptina y es otro regulador de STAT3 que está comprometido en este estado. Esta drástica modificación en la señalización periférica también se convierte en un blanco terapéutico ${ }^{(32)}$. Finalmente, esta variación en la retroalimentación explica porqué los niveles muy elevados de leptina se comportan como la deficiencia relativa de la misma, esta conclusión se podría complementar con los hallazgos de Franek et at. que muestran que las concentraciones de leptina en pacientes obesos podrían, además, ser independientes y correlacionarse negativamente con la densidad mineral ósea (DMO) en el sexo masculino pero positivamente en el sexo femenino que presentan alelos específicos del gen de la leptina $(-2548 \mathrm{~A})^{(33)}$.

\section{Relación entre obesidad y hueso}

La obesidad es una enfermedad de gran impacto para la OMS, puesto que es una de las diez primeras causas de muerte en el mundo, con un alto número de afectados, como lo muestra en su comunicado del 2008, según el cual 1.400 millones de adultos tenían sobrepeso y de éstos más de 200 millones de hombres y 300 millones de mujeres eran obesas ${ }^{(34)}$. En Latinoamérica, la obesidad creció en $40 \%$ entre el 2002 y el 2010, con un gran impacto sobre la mortalidad total. En su declaración, la OMS muestra que al menos 2,8 millones de personas adultas mueren como consecuencia de obesidad o sobrepeso ${ }^{(34)}$. No alejados de estas cifras, para el 2007, según la Encuesta Nacional de Salud, el sobrepeso y la obesidad en Colombia representaban el $32 \%$ y $13 \%$, respectivamente, con diferencias entre regiones, siendo más prevalente en la Orinoquía y el Amazonas con un 20\% de prevalencia para obesidad ${ }^{(35)}$. En el 2010, la Encuestra Nacional de Demografía y Salud (ENDS) informó que el porcentaje de sobrepeso y obesidad había aumentado, llegando a un $47 \%$ en la población general ${ }^{(36)}$, lo que refleja una tendencia creciente, tanto en la incidencia como en la prevalencia de esta patología, que genera un gran deterioro del estado de salud general, disminución en la expectativa de vida y aumento de los costos en salud ${ }^{(37)}$.

Es clara la literatura existente que relaciona la obesidad con el aumento del riesgo cardiovascular, cerebrovascular, así como el desarrollo de hepatopatías, nefropatías, endocrinopatías, alteraciones sexuales, alteraciones neurológicas y articulares, principalmente. Claramente, la obesidad afecta deleté- reamente todo órgano y sistema en el cuerpo humano ${ }^{(38)}$, pero curiosamente existe evidencia contradictoria en cuanto a la relación entre la obesidad y la densidad mineral ósea (DMO), considerada por algunos benéfica para el funcionamiento del hueso como órgano dinámico ${ }^{(39,40)}$. Entre ellos, resalta Mullingan et al., quienes evidenciaron en su estudio en adultos sanos el aumento de la masa grasa asociada con incremento de la $\mathrm{DMO}^{(41)}$, mientras que otros siguen considerándola contraproducente, puesto que el aumento de peso lleva como respuesta a un aumento de los niveles de leptina en sangre, que inicialmente se traducen en un balance energético negativo (pérdida de grasa), esto en pacientes previamente con peso normal. En el paciente obeso, este mecanismo se afecta, perdiendo la capacidad anorexígena y de igual forma su respuesta metabólica, favoreciendo un balance energético positivo ${ }^{(42)}$. La concentración de leptina refleja la reserva de energía en la masa grasa y también refleja la cantidad de grasa corporal ${ }^{(43)}$. En el estudio NHANES III se evidencia la relación positiva entre IMC y la hiperleptinemia ${ }^{(44)}$ lo que fortalece esta relación bioquímica como respuesta anormal en la obesidad.

Para entender adecuadamente la relación entre la obesidad y el hueso, debemos tocar aspectos de la fisiología del tejido adiposo como órgano endocrino, en donde los principales protagonistas son las adipocinas, con funciones bien establecidas a nivel paracrino, autocrino y endocrino ${ }^{(45)}$.

Entendiendo la obesidad a nivel celular como un estado proinflamatorio, las citocinas más relevantes para explicar el ambiente proinflamatorio son: leptina, adiponectina, factor de necrosis tumoral alfa (TNF- $\alpha$ ) y la interleucina 6 (Il-6) ${ }^{(46)}$. Cada una de ellas tiene su función específica y con una interacción clara en cada órgano que afectan. Por nuestro interés, es la leptina en donde fijaremos nuestra atención al ser esta adipocina el eje central del efecto deletéreo a nivel óseo.

La leptina es considerada como una citocina proinflamatoria, pertenece a la familia de las citocinas de tipo IL-6 ${ }^{(46,47)}$. Con múltiples funciones a nivel circulatorio, metabólico, reproductivo y en la regulación del balance de energía, el cual se lleva a cabo a nivel central a través del hipotálamo, disminuyendo la ingesta de alimentos, aumentando el gasto energético y estimulando la lipólisis ${ }^{(39,48)}$. Pero, además de esto, a nivel óseo presenta un efecto netamente protector, en individuos con peso normal, interviniendo en el metabolismo óseo, al regular la formación cortical, favoreciendo la activación de la vía simpática ${ }^{(40)}$. De igual forma, a nivel medular, favorece la formación de osteoblastos, actuando directamente en la diferenciación celular ${ }^{(6)}$. Además, a nivel de la médula ósea favorece la producción de osteoblastos y de IGF-1, estimulando también la proliferación de osteoblastos precursores que favorecen la resistencia a la apoptosis. Asimismo el aumento de la leptina, incrementa la expresión de osteoprotegerina (OPG), lo que disminuye la activación del receptor activador de RANK ligando ${ }^{(41,43,48)}$. 
En contraposición a lo anterior, en sujetos obesos se describen acciones deletéreas como la regulación de la expresión de neuromedina $U$, la cual puede disminuir la masa tanto cortical como trabecular ${ }^{(49)}$. Otros mecanismos de acción de la leptina en el hueso que generan deterioro de la masa ósea, son: la disminución en receptores de serotonina 2, la cual en estudios con ratones knockout causó disminución de la masa trabecular en vértebras y tibia ${ }^{(43,50)}$. De igual forma, se evidencia que el incremento del neuropéptido Y (NPY) redujo la masa ósea, al favorecer el incremento de los niveles de factor inhibidor de osteoblastos hipotalámico, el cual reduce la habilidad de la matriz ósea para la formación de osteoblastos, en estados de resistencia a la leptina. Esta retroalimentación negativa de la leptina sobre el NPY se ve afectada, favoreciendo indirectamente su efecto deletéreo sobre el hueso ${ }^{(29)}$. También está establecida la relación negativa entre leptina, testosterona y su fracción biodisponible, que genera una disminución de la función protectora de la testosterona sobre la $\mathrm{DMO}^{(44)}$. Cao et al., mostraron en su estudio cómo las dietas ricas en grasas, generan un aumento de la leptina y una disminución en el hueso trabecular(51), lo que se explica por la intervención en el metabolismo óseo de la leptina, generando regulación negativa de los precursores mesenquimales en la diferenciación celular, disminuyendo la osteoblastogénesis y favoreciendo la adipogénesis a nivel medular, lo que influye negativamente en la $\mathrm{DMO}^{(52)}$.

Los datos mencionados anteriormente buscan exponer desde un enfoque fisiopatológico la relación existente entre la leptina y la DMO, con puntos contundentes de interrelación bioquímica que explican el papel de cada uno de los órganos $\mathrm{y}$ sus productos para mantener una homeostasis funcional. A partir de los argumentos previamente expuestos se concluye, desde el punto de vista bioquímico, cómo el exceso de adipocinas determina un riesgo para la salud ósea, pues genera una alteración en la formación de los osteoblastos y además favore la adipogénesis medular, convirtiéndose en un factor de riesgo adicional en la población con baja DMO. Si esto lo llevamos a una población obesa con sarcopenia (obesidad sarcopénica), donde la unidad funcional hueso-músculo está ya alterada, tener hiperleptinemia como marcador de seguimiento podría ser una herramienta en la práctica clínica de gran ayuda, puesto que cada vez se esclarece más la gran importancia de la leptina como marcador protagónico, sobre todo en riesgo cardiovascular en línea con lo establecido por Batsis et $\mathrm{al}^{(54)}$. En el Estudio nacional de salud y evaluación nutricional III, publicado por la Mayo Clinic en el 2015, luego de un seguimiento de más de siete años de una población obesa masculina, se logró establecer que los niveles altos de leptina son un predictor de mortalidad cardiovascular. Es evidente que para poder establecer esto se requieren estudios a largo plazo donde se logre evaluar estos dos indicadores y así validar este marcador sérico.

Debemos también mencionar que en la literatura mundial la evidencia existente entre el IMC y DMO muestra una relación directa, que favorece la salud ósea ${ }^{(53)}$, esto en población con sobrepeso y obesidad grado I, lo que sería contradictorio con lo enunciado anteriormente. Por tal motivo, podríamos concluir que existe un efecto inicial dinámico en el hueso que es promovido por las fuerzas que generan el exceso de peso sobre el hueso, pero este estímulo es temporal y la perpetuidad en la sobrecarga del hueso lleva, a largo plazo a que se altere el equilibrio, favoreciendo que el efecto metabólico de las adipocinas tome el control y lleve a un gasto óseo mayor de forma crónica.

Lo anterior debe llevar a cuestionarnos qué estrategias podremos usar en la práctica clínica para abordar a nuestros pacientes con problemas de peso, entendiendo que la obesidad no es un problema reflejado en una báscula sino, por el contrario, es una red de alteraciones bioquímicas que afectan a corto y largo plazo al ser humano, de ahí que todas las ayudas diagnósticas para prevenir los desenlaces negativos de esta enfermedad deben ser usadas en nuestros pacientes para ofrecer todo el arsenal terapéutico y no terapéutico para combatir este flagelo, y no optar por dieta como solución única a todas las preguntas generadas.

\section{Referencias}

1. Iwaniec UT, Dube MG, Boghossian S, Song H, Helferich WG, Turner RT, et al. Body mass influences cortical bone mass independent of leptin signaling. Bone. 2009;44(3):404-12. Epub 2008/12/20.

2. Tartaglia LA, Dembski M, Weng X, Deng N, Culpepper J, Devos R, et al. Identification and expression cloning of a leptin receptor, OB-R. Cell. 1995;83(7):1263-71. Epub 1995/12/29.

3. Farooqi IS, Jebb SA, Langmack G, Lawrence E, Cheetham $\mathrm{CH}$, Prentice AM, et al. Effects of recombinant leptin therapy in a child with congenital leptin deficiency. The New England journal of medicine. 1999;341(12):879-84. Epub 1999/09/16.

4. Hebebrand J, Muller TD, Holtkamp K, Herpertz-Dahlmann B. The role of leptin in anorexia nervosa: clinical implications. Molecular psychiatry. 2007;12(1):23-35. Epub 2006/10/25.

5. Pasco JA, Henry MJ, Kotowicz MA, Collier GR, Ball MJ, Ugoni AM, et al. Serum leptin levels are associated with bone mass in nonobese women. The Journal of clinical endocrinology and metabolism. 2001;86(5):1884-7. Epub 2001/05/10.

6. Friedman JM. Leptin, leptin receptors, and the control of body weight Nutrition reviews. 1998;56(2 Pt 2):s38-46; discussion s54-75. Epub 1998/05/02.

7. Dardeno TA, Chou SH, Moon HS, Chamberland JP, Fiorenza CG, Mantzoros CS. Leptin in human physiology and therapeutics. Frontiers in neuroendocrinology. 2010;31(3):377-93. Epub 2010/07/06.

8. Simón E DBAS. Leptina y obesidad. Anales del sistema sanitario de Navarra. 2002;25:53-64.

9. Considine RV, Sinha MK, Heiman ML, Kriauciunas A, Stephens TW, Nyce MR, et al. Serum immunoreactive-leptin concentrations in normal-weight and obese humans. The New England journal of medicine. 1996;334(5):292-5. Epub 1996/02/01.

10. Chan JL, Heist K, DePaoli AM, Veldhuis JD, Mantzoros CS. The role of fall- 
ing leptin levels in the neuroendocrine and metabolic adaptation to short-term starvation in healthy men. The Journal of clinical investigation. 2003;111(9):1409-21. Epub 2003/05/03.

11. Bjorbaek C, Elmquist JK, Michl P, Ahima RS, van Bueren A, McCall AL, et al. Expression of leptin receptor isoforms in rat brain microvessels. Endocrinology. 1998;139(8):3485-91. Epub 1998/07/29.

12. Ernst MB, Wunderlich CM, Hess S, Paehler M, Mesaros A, Koralov SB, et al. Enhanced Stat3 activation in POMC neurons provokes negative feedback inhibition of leptin and insulin signaling in obesity. The Journal of neuroscience : the official journal of the Society for Neuroscience. 2009;29(37):11582-93. Epub 2009/09/18.

13. Gong L, Yao F, Hockman K, Heng HH, Morton GJ, Takeda K, et al. Signal transducer and activator of transcription-3 is required in hypothalamic agoutirelated protein/neuropeptide $\mathrm{Y}$ neurons for normal energy homeostasis. Endocrinology. 2008;149(7):3346-54. Epub 2008/04/12.

14. Santillán Benítez JG, Mendieta Zerón H, Ordóñez Quiroz Á, LM. GO. La leptina en la carcinogénesis mamaria. Vías de señalización. Química Viva. 2012;11(2):91-111.

15. Morrison CD. Leptin signaling in brain: A link between nutrition and cognition? Biochimica et biophysica acta. 2009;1792(5):401-8. Epub 2009/01/10.

16. Harvey J, McKay NG, Walker KS, Van der Kaay J, Downes CP, Ashford ML. Essential role of phosphoinositide 3-kinase in leptin-induced K(ATP) channel activation in the rat CRI-G1 insulinoma cell line. The Journal of biological chemistry. 2000;275(7):4660-9. Epub 2000/02/15.

17. Plum L, Ma X, Hampel B, Balthasar N, Coppari R, Munzberg H, et al. Enhanced PIP3 signaling in POMC neurons causes KATP channel activation and leads to diet-sensitive obesity. The Journal of clinical investigation. 2006;116(7):1886-901. Epub 2006/06/24.

18. Bjorbaek C, Buchholz RM, Davis SM, Bates SH, Pierroz DD, Gu H, et al. Divergent roles of SHP-2 in ERK activation by leptin receptors. The Journal of biological chemistry. 2001;276(7):4747-55. Epub 2000/11/22.

19. Rahmouni K, Sigmund CD, Haynes WG, Mark AL. Hypothalamic ERK mediates the anorectic and thermogenic sympathetic effects of leptin. Diabetes. 2009;58(3):536-42. Epub 2008/12/11.

20. Kim GS, Hong JS, Kim SW, Koh JM, An CS, Choi JY, et al. Leptin induces apoptosis via ERK/cPLA2/cytochrome c pathway in human bone marrow stromal cells. The Journal of biological chemistry. 2003;278(24):21920-9. Epub 2003/04/01.

21. Fruhbeck G. Intracellular signalling pathways activated by leptin. The Biochemical journal. 2006;393(Pt 1):7-20. Epub 2005/12/13.

22. ME. FVV. Señalización de la leptina. Revista de Educación Bioquímica. 2006;25(2):50-4.

23. Hamrick MW. Leptin and bone: A consensus emerging? IBMS BoneKEy BoneKEy-Osteovision. 2007;4(3):99-107.

24. Rosen CJ, Bouxsein ML. Mechanisms of disease: is osteoporosis the obesity of bone? Nature clinical practice Rheumatology. 2006;2(1):35-43. Epub 2006/08/26.

25. Wren AM, Small CJ, Abbott CR, Jethwa PH, Kennedy AR, Murphy KG, et al. Hypothalamic actions of neuromedin U. Endocrinology. 2002;143(11):422734. Epub 2002/10/26

26. Yadav VK, Oury F, Suda N, Liu ZW, Gao XB, Confavreux C, et al. A serotonin-dependent mechanism explains the leptin regulation of bone mass, appetite, and energy expenditure. Cell. 2009;138(5):976-89. Epub 2009/09/10.

27. Hamrick MW, Della-Fera MA, Choi YH, Pennington C, Hartzell D, Baile CA. Leptin treatment induces loss of bone marrow adipocytes and increases bone formation in leptin-deficient ob/ob mice. Journal of bone and mineral research : the official journal of the American Society for Bone and Mineral Research. 2005;20(6):994-1001. Epub 2005/05/11.

28. Holloway WR, Collier FM, Aitken CJ, Myers DE, Hodge JM, Malakellis M, et al. Leptin inhibits osteoclast generation. Journal of bone and mineral research : the official journal of the American Society for Bone and Mineral Research. 2002;17(2):200-9. Epub 2002/01/29.

29. Cirmanová V, Bayer M, Stárka L, Zajícková K. The effect of leptin on Bone: An Evolving Concept of Action. 2008; 57 (Suppl. 1): 143-51. physiological research-ISSN 0865-8408

30. Burguera B, Couce ME, Curran GL, Jensen MD, Lloyd RV, Cleary MP, et al. Obesity is associated with a decreased leptin transport across the bloodbrain barrier in rats. Diabetes. 2000;49(7):1219-23. Epub 2000/07/26.

31. Oswal A, Yeo G. Leptin and the control of body weight: a review of its diverse central targets, signaling mechanisms, and role in the pathogenesis of obesity. Obesity (Silver Spring). 2010;18(2):221-9. Epub 2009/08/01.

32. Turner RT, Kalra SP, Wong CP, Philbrick KA, Lindenmaier LB, Boghossian $\mathrm{S}$, et al. Peripheral leptin regulates bone formation. Journal of bone and mineral research : the official journal of the American Society for Bone and Mineral Research. 2013;28(1):22-34. Epub 2012/08/14.

33. Franek E, Nowak J, Safranow K, Adler G, Binczak-Kuleta A, Ciechanowicz A et al. G(-2548)A leptin gene polymorphism in obese subjects is associated with serum leptin concentration and bone mass. Polskie Archiwum Medycyny Wewnetrznej. 2010;120(5):175-80. Epub 2010/05/27.

34. OMS. Nota descriptiva $\mathrm{N}^{\circ} 311$. Obesidad y sobrepeso. Disponibleen: http:// www.who.int/mediacentre/factsheets/fs311/es/index.html

35. Ministerio de la Protección Social. Encuesta Nacional de Salud, 2007.

36. Erazo M. Visión global en relación a la obesidad. Rev. Med. Clin, Condes. 2012; 23(2) 196-200

37. Acosta, K. La obesidad y su concentración según nivel socioeconómico en Colombia. Num 170, Julio, 2012.

38. Aranceta, j. Foz, M. Gil B, Jover, E. Mantilla, T. Millán, J. Monereo, S. Moreno, B. Documento de consenso: obesidad y riesgo cardiovascular. Clin Invest Arterioscl 2003;15(5):196-233

39. Papapietro, K, Massardo, T. Riffo, A. Díaz, E. Araya V, Adjemian, D. Montesinos, G. Castro, G. Disminución de masa ósea post-cirugía bariátrica con by-pass en Y de Roux. Nutr Hosp. 2013;28:631-636. DOI:10.3305/ nh.2013.28.3.6400

40. Maor, G. Rochweger, M. Sege, Y. Phillips M. Leptin Acts as a Growth Factor on the Chondrocytes of Skeletal Growth Centers, Journal of bone and mineral research, Volume 17, Number 6, 2002

41. Rao, M. Schambelan, M. Tai, V. Abrams, D. Khatami, H. Havel, P. Sakkas, G. Mulligan,K. Assessing the Association between Leptin and Bone Mineral Density in HIV-InfectedMen. Hindawi Publishing Corporation AIDS Research and Treatment Volume 2012, Article ID 103072, 5 pages doi:10.1155/2012/103072

42. Friedman, J. Halaas, J. Leptin and the regulation of body weight in mammals, NATURE | VOL 395 | 22 OCTOBER 1998 | www.nature.com

43. Legiran, S. Brandi, M. Bone mass regulation of leptin and postmenopausal osteoporosis with obesity. Clinical Cases in Mineral and Bone Metabolism 2012; 9(3): 145-149

44. Anagnostis,P. Vakalopoulou,S. Charizopoulou, M. Kazantzidou, E. Chrysopoulou, T. Moka,E. Agapidou, A. Zournatzi, V. Garipidou, V. Is there any association between leptin levels and bone mineral density in haemophiliac men? Arch Med Sci 2013; 9, 3: 459-465

45. Coelho, M. Oliveira, T. Fernandes, R. Biochemistry of adipose tissue: an endocrine organ, Arch Med Sci 2, April / 2013

46. Kwon, H. Pessin, J. Adipokines mediate inflammation and insulin resistance, Front Endocrinol (Lausanne). 2013; 4: 71

47. Coelho, M. Oliveira, T. Fernandes, R. Biochemistry of adipose tissue: an endocrine organ. Arch Med Sci 2013; 9, 2: 191-200 DOI: 10.5114/ aoms.2013.33181

48. Lazarenko OP, Rzonca SO, Suva LJ, Lecka-Czernik B. Netoglitazone is a PPARgamma ligand with selective effects on bone and fat. Bone. 2006;38(1):7484. Epub 2005/09/03.

49. Mantzoros, C. Magkos, F. Brinkoetter, M. Sienkiewicz, E. Dardeno, T. Kim, S. Hamnvik, OP. Koniaris, A. Leptin in human physiology and pathophysiology. Am J Physiol Endocrinol Metab 301: E567-E584, 2011.

50. Motyla, K. Rosena, C. Understanding leptin-dependent regulation of skeletal homeostasis, Biochimie. 2012 October; 94(10): 2089-2096. doi:10.1016/j biochi.2012.04.015

51. Cao JJ, Gregoire BR, Gao H. High-fat diet decreases cancellous bone mass but has no effect on cortical bone mass in the tibia in mice. Bone. 2009;44(6):1097-104. Epub 2009/03/07.

52. Scheller, E. Song, J. Dishowitz, M. Hankenson,K. Krebsbac, K. A potential role for the myeloid lineage in leptin-regulated bone Metabolism. Horm Metab Res. 2012 January ; 44(1): 1-5. doi:10.1055/s-0031-1297971

53. Zhao, L. Jiang, H. Papasian, C. Maulik, D. Drees, B. Hamilton, J. Deng, H. Correlation of Obesity and Osteoporosis: Effect of Fat Mass on the Determination of Osteoporosis. Journal of bone and mineral research. Volume 23, Number 1,2008

54. Batsis, J. Sahakyan, K. Singh, P. Bartels, S. Somer, V. Lopez-Jimenez, F. Leptin, Adiposity, and Mortality: Results from the National Health and Nutrition Examination Survey III, 1988 to 1994. Mayo Clinic Proceeding. 2015:90(4):481-491. 